\title{
Human Localization based on Distributed Laser Range Finders
}

\author{
Peng Duan, Guohui Tian* and Wei Zhang \\ School of Control Science and Engineering, \\ Shandong University, Jinan, 250061, P.R. China \\ *Corresponding author: g.h.tian@sdu.edu.cn
}

\begin{abstract}
Localizing humans inside a home or office environment is vital for various service robot applications. This paper presents a novel method to estimate human locations using distributed laser range finders. First, the Kalman filter is employed on the laser data to produce a statistically optimal scan estimate. Next, Scan points in the foreground are then grouped using mean shift clustering algorithm. An area-dlvided clustering node is introduced to ensure enough clusters to represent the contour of human. Finolly, the centers of humans are estimated from the obtained clusters using incomplete ellipse fiting. Experiments are conducted to prove the robustness and efficiency of the proposed method.
\end{abstract}

Keywords: Human localization, Kalman filter, Mean Shift clustering, distributed laser range finders

\section{Introduction}

Recently, the research on service robot has received a lot of attention over the last decade $[1,2]$. Some robots are suceessfully working at home and in office to provide services. In general, to complete a service task autonomously, the robot has to focus its attention on humans and aware of their presence. There are various scenarios where tracking people in a family environment or office spaces is essential to many real life applications [2], such as instructing a service robot to deliver drinks, the position of people must be know in advance. Therefore, it is necessary to haye a human localization system that returns people's current positions.

However, human localization is a very challenging task, as human behaviors are often unpredictable. Researchers have been used different methods to deal with this problem and many systems have been developed over the years. These systems employed many kinds of sensors such as cameras, sensor networks, infrared bearing sensor and passive RFID-system. Cameras are easy to install and have frequently been employed for indoor localization. For example, Shen et al. [3] introduced a multiple cameras localization algorithm in a cluttered space, and the error is within $40 \mathrm{~cm}$. Similarly, Yu et al. [4] developed a human localization system for smart home via multi-camera and sensory floor approaches. By integrating information from sensory floor, the localization results are more reliable. Yao and Odobez [5] presented an algorithm in a Bayesian framework for the tracking of a variable number of 3D persons to deal with partial field-of-view overlap. However, it is difficult to develop a robust system using cameras because of the illumination and resolution problems. Obo et al. [6] contributed at proposing a human localization method in sensor networks to monitor elderly people, which apply a spiking neural network to extract feature points for human localization. Data communication becomes the bottleneck of localization accuracy. In addition, Yang et al. 
[7] introduced a method using a compressed infrared bearing sensor for human localization as infrared detectors are sensitive to the change of infrared radiation induced by human motions. In [8], an approach for human localization using a passive UHF RFID was presented. The idea is to characterize the change of RSSI caused by the presence of a person inside an RFID augmented deployment area. The complexity of indoor RF-propagation makes localization a challenging task in passive RFID systems. Especially in indoor environments, fixtures, fittings and also human can cause reflections, diffractions and absorptions of radio signals. While laser range finder is able to perform high-accuracy measurement, and successfully used in some challenging vision tasks. For example, Yao et al. [9] used a laser-based system to identify cylinders through exploring the 3D frontal surface. Kim et al. [10] proposed a method for people tracking using a robot in motion with laser range finder. Similar applications appeared in [11]. However, one laser range finder on board is helpless for multiple people localization especially in the dynamic environment. But the problem above can be effectively solved if we use several laser range finders based on-collaborative perception. Adam et al [12] proposed and evaluated a detection-based algorithm for tracking a variable number of dynamic targets online using multi-laser range finders. In [13], a system to detect and track of moving objects at an intersection using a network of horizontal laser range finders is presented. Both of the two systems above have achieved encouraging results.

The aim of this paper is to localize multiple people using distributed laser range finders in indoor environment. In consideration of the scan beams are spaced quite far apart over a long distance, the detection of larger targets, like a forso, is morecrobust than detection of small features like legs. The main challenges of luman localization are the way to represent the detection characteristics and the valid approach of extrating human center positions.

The remainder of this paper is organized as follows. Section 2 gives discussions about statistic characteristic of laser data and introduces laser data processing using Kalman filter.

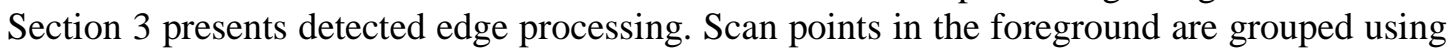
mean shift clustering algorithm, along with an area-divided clustering mode is introduced. This section is followed by Mntroduction of cooperative localization system in Section 4. In this section sensors position ealibration and human center position extraction were discussed. Experimental results ate given in Section 5. Finally, we conclude the paper in Section 6.

\section{Data Processing based on the Kalman Filtering}

In this section, laser dala processing using Kalman filter is introduced. The laser range finder we used is Hokuyo UTM-30LX, which scans at $40 \mathrm{~Hz}$ with the angular resolution of $0.25^{\circ}$ The effective measurement distance is from 0.1 to $30 \mathrm{~m}$. As a rule of thumb, sensors are subject to noise. Although this uncertainty may be very small for most kinds of laser range finders and it even can be neglected comparing with other measurement equipments, to model the noise in sensor measurements accurately, laser data analysis is put on the agenda.

\section{Statistic characteristic of laser data}

As our spatial constraints, the data can hardly achieve the max-range measurement. So our laser range finder observation model can be expressed as:

$$
z\left(s_{t}, \theta_{t}\right)=d\left(s_{t}, \theta_{t}\right)+\varepsilon_{d, t}
$$

where $d\left(s_{t}, \theta_{t}\right)$ is an ideal observation model and $\varepsilon_{d, t}$ may be a certain distribution of noise. Our main aim is to determine the noise distribution characteristic. We randomly chose several laser beams. Each of beams is measured for 1004 times to observe the distribution of the laser data. Details results are shown in Figure 1 (a)-(f). 


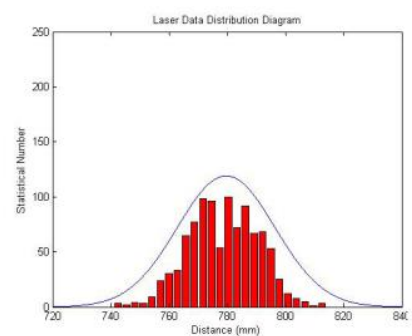

(a)

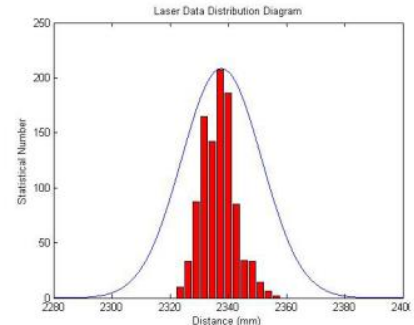

(d)

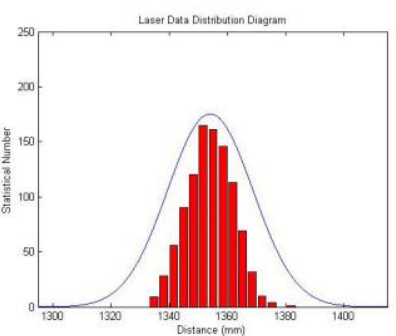

(b)

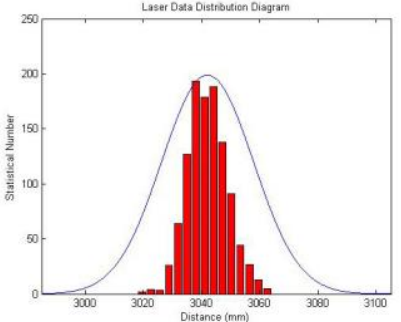

(e)

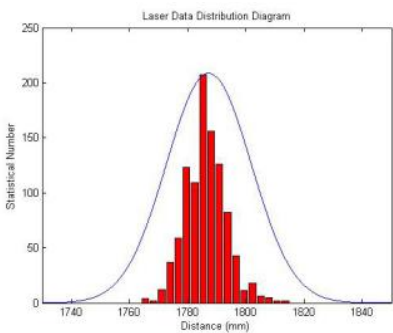

(c)

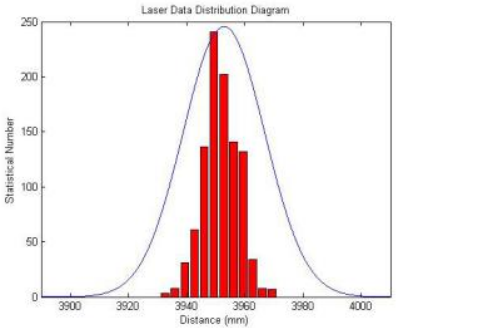

(f)

Figure 1. Distributions of laser data at different distances. (a) $780 \mathrm{~mm}$. (b) 1360 mm. (c) $1790 \mathrm{~mm}$. (d) $2340 \mathrm{~mm}$. (e) $3040 \mathrm{~mm}$. (f) $3950 \mathrm{~mm}$.

Figure 1 shows the distributions of laser datadt different distances. For these small figures, they have the same coordinate system. The horizontal axis is the distance between laser range finder and obstacles, and the vertical axisis the number of statistics. Although we can hardly find an accurate way to measure the actual distance from the laser to the obstacles, the distribution of laser data is obvious Fortunately, the statistical results show that the distribution property of the data is obeyed to Caussian. Figure 2 shows some other statistical characteristics of the data in Figure 1. The difference between the mean and the maximum or minimum of laser data is shown in Figure 2(a). Figure 2(b) illustrates the changing trend of variance.

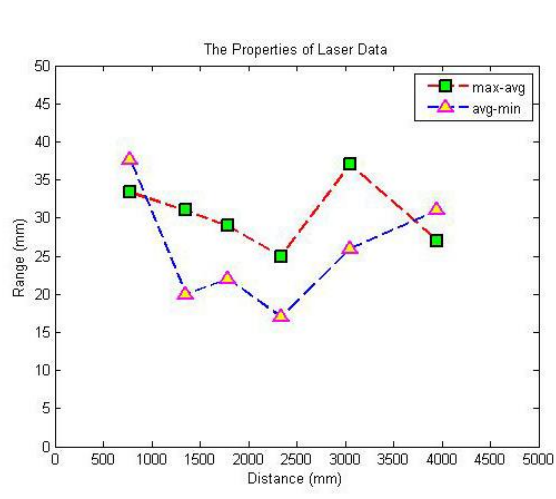

(a)

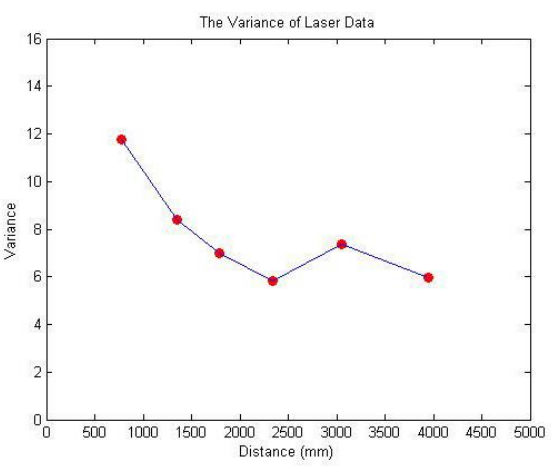

(b)

Figure 2. Statistical characteristics of laser data in Figure 1. (a) Difference between the mean and the maximum or minimum data. (b) The Changing trend of variance.

\subsection{Kalman Filtering}

As discussed above, the noise of the data is obeyed to Gaussian distribution that accords with the assumption of Kalman filters. So a Kalman filter is employed for denosing. The Kalman filter is a recursive filter which is invented known as a technique for filtering and prediction in linear systems. Since that time, due in large part to 
advances in digital computing, the Kalman filter has been the subject of extensive research and applications from radar tracking to computer vision [14]. The filtering algorithm has just to store the last state parameters to estimate the next state. This makes it interesting for real-time applications. The kalman filtering algorithm estimates the process state at some time and then obtains feedback in the form of measurements. It can be divided into two groups: time update equations and measurement update equations. The time update equations of discrete Kalman filter are presented below:

$$
\left\{\begin{array}{l}
X_{k \mid k-1}=A_{k} X_{k-1 \mid k-1}+B u_{k} \\
P_{k \mid k-1}=A_{k} P_{k-1 \mid k-1} A_{k}^{T}+Q_{k}
\end{array}\right.
$$

The two equations above project the state and covariance estimates from the step $k-1$ to step $\mathrm{k}$, where $X_{k \mid k-1}$ is a priori state estimated from $X_{k-1 \mid k-l}$ at step k-1 and $u_{k}$ is the input of discretetime controlled process. $A_{k}$ is the state transition matrix and $B$ denotes the control input matrix. $P_{k \mid k-1}$ is a priori error covariance generated from $X_{k-1 \mid k-1}$ at step $k-1$ and $Q_{k}$ is the covariance of discrete-time controlled process. The measurement update equations of discrete Kalman filter are described as follow:

$$
\left\{\begin{array}{l}
K_{k}=P_{k \mid k-1} H_{k}^{T}\left(H_{k} P_{k \mid k} H_{k}^{T}+R_{k}\right)^{-1} \\
X_{k \mid k}=X_{k \mid k-1}+K_{k}\left(Z_{k}-H_{k} X_{k \mid k-1}\right) \\
P_{k \mid k}=\left(I-K_{k} H_{k}\right) P_{k \mid k-1}
\end{array}\right.
$$

The first task during the measurement update is to compute the Kalman gain $K_{k}$, where $H_{k}$ is the measurement matrix and $R_{k}$ describes the covarlance of measurement process. The next step is to actually measure the procêss obtain $Z_{\text {. }}$ and then to generate a posteriori state estimate $X_{k \mid k}$ by incorporating the measurement. The final step is to obtain a posteriori error covariance estimate $P_{k \mid k}$. After each time and measurement update pair, the process is repeated with the previous (a posteriori) estimates used to predict the new (a priori) estimates.

The result of data processing illustrates the effectiveness of the Kalman filter as shown in Figure 3. Figure 3(a)shows the raw data along with the error about $6 \mathrm{~cm}$. The filtered data is shown in Figure 3(b) and the error is within $2 \mathrm{~cm}$.

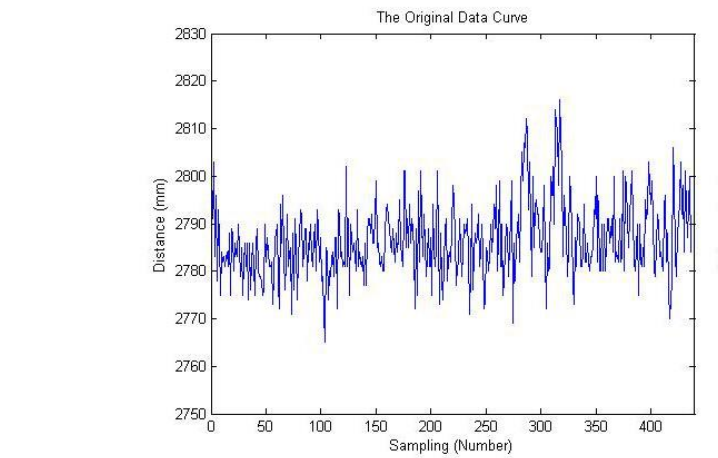

(a)

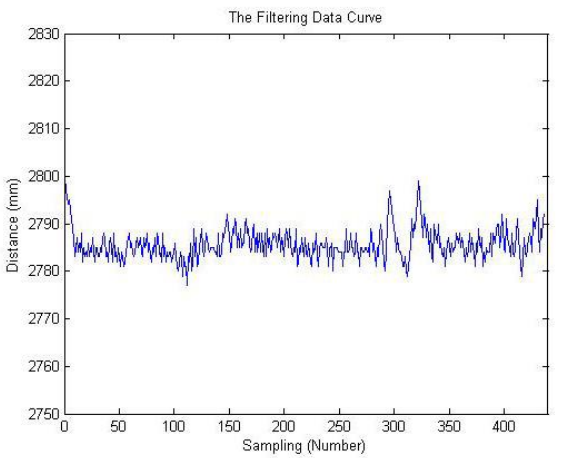

(b)

Figure 3. Result of data processing using Kalman filter. (a) The raw data. (b) The filtered data.

The filtering process appears to be satisfactory. However, an undesirable phenomenon appeared as shown in Figure 4. Figure 4(a) illustrates the raw data along with abrupt change. Abrupt change in data for one laser beam is a very common phenomenon in our localization system. The position of a person is dynamic, whereas one laser beam may hit on human body 
at one sampling time and the next sampling time may be not. Figure 4(b) shows the filtered data. Unfortunately, the filtered data cannot track to the measurement tightly. The tracking efficiency of filter depends on the sampling frequency and Kalman filter parameters. Due to the sampling frequency is a constant for our equipment. The parameters setting of Kalman filter is particularly important. There are two parameters that may contribute to inefficient process. One is the measurement variance $R_{k}$. If $R_{k}$ is too great, the filter will be "slowly" to believe the measurements. The tracking efficiency is disappointed. If $R_{k}$ is small, the filter will be "quickly" and "firmly" to believe the measurements. However, the role of filter will be reduced as $R_{k}$ is too large. We would expect the best performance in terms of balancing responsiveness and estimate variance $R_{k}$. So just adjusting the value of $R_{k}$ is not a good idea for Kalman filter in order to solve the problem of the abrupt change of the data.

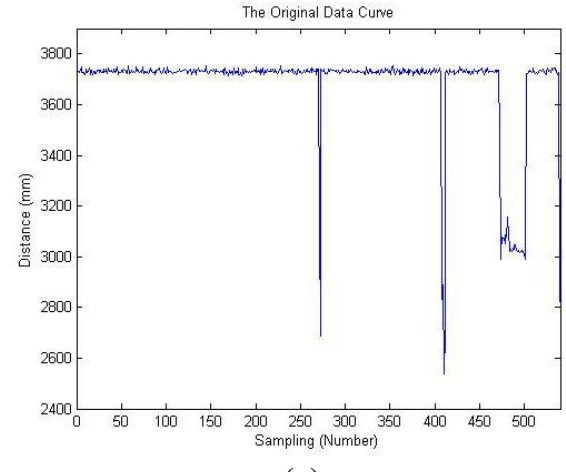

(a)

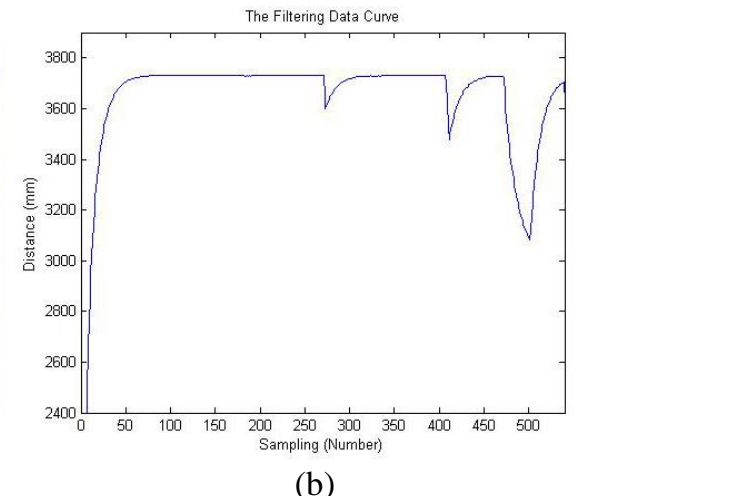

(b)

Figure 4 An undesirable result of data processing. (a) Raw data of one beam with abrupt change. (b) The matching filtered result.

Another parameter is the accuracy of prediction in the time update equations of Kalman filter. That means the parameter $A_{k}$ in the Equation (2) is very important. In our filtering experiments, we let $A_{k}$ equals to an unit matrix and the result is unsatisfactory. Because it is unable to predict one data will increasse or decrease due to the change of obstacles in the dynamic environment It is more difficult to predict the value of data next time from the current data for one beam that is changing suddenly. To solve this problem, we set a threshold. The threshold is selected according to the statistical characteristic of data. Therefore, the parameter $a_{k}$ in the Equation (2) becomes:

$$
\mathcal{A}_{k}= \begin{cases}0 & \text { if }\left|Z_{k}-X_{k-1 \mid k-1}\right| \leq \text { threshold } ; \\ I & \text { else } ;\end{cases}
$$

If the difference between the measurement $Z_{k}$ and previous posteriori estimate $X_{k-1 \mid k-1}$ is larger than the threshold, let $A_{k}$ equals to zero matrix and set $X_{k \mid k-1}$ equals to $Z_{k}$. That means our filter is more inclined to believe the measurements changing suddenly than the data changing slowly that may contains error. Figure 5 illustrates the result of using an improved parameter to process these abnormal data. Then we received relatively accurate and stable filtered data. 


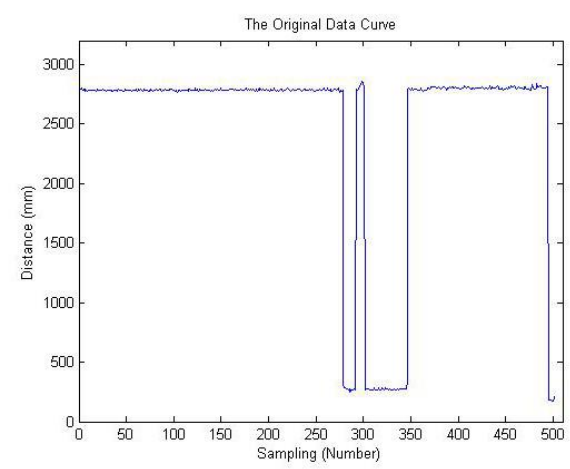

(a)

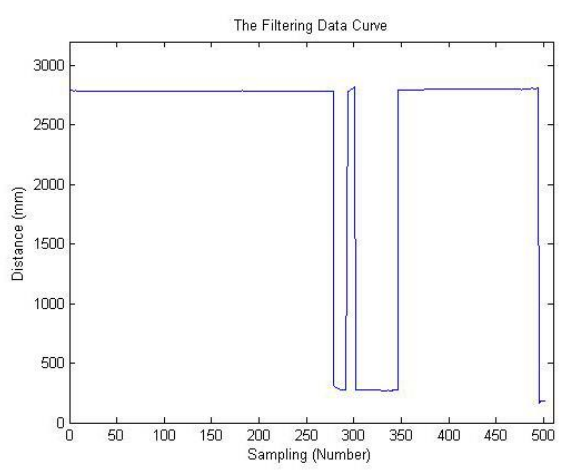

(b)

Figure 5. Result of data processing using an improved parameter. (a) Raw data of one beam with abrupt change. (b) The matching filtered result

\section{Clustering of Detected Edge Points}

The major problem of using laser range finder for human localization is that data from laser range finder is only distances and angles to object surfaces. Before designing our localization system, we should observe human detection features under laser range finder primarily as shown in Figure 6. Figure 6(a)illustrates a delection contour of one person facing the detecting equipment and Figure 6(b) displays the side contour of human trunk toward the laser range finder.
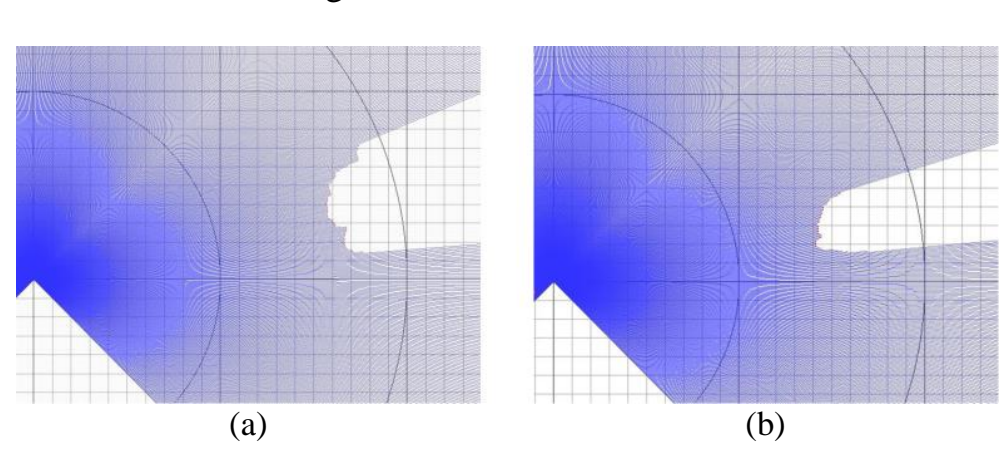

(b)

Figure 6 Detected feature using laser range finder. (a) Result of one person facing detection equipment. (b) Side contour of human trunk toward laser range finder.

So in order to estimate the human center position, we need to explore an appropriate way to describe the detection contour. Therefore, we transform distances and angles into coordinate point Using detected points to represent human contour is good pattern. However, there is no need to use a large number of edge points to represent the detection characteristics. Instead, we can choose some of points for representation. A better way is using clustering [15]. Many clustering algorithms are regarded as means for optimizing certain measures about the portioning. For example, the $k$-means clustering algorithm is aiming at minimizing the within-group sum of squared errors [16], and the maximum entropy clustering algorithm is to maximize entropy while the within-group sum of squared errors is held constant [17]. Here, we used a simple iterative procedure algorithm named Mean Shift. This algorithm shifts each data point to the average of data points in its neighborhood to characterize the points. Cheng [18] has proved that a specific weight function in mean shift could unify certain fuzzy clustering algorithms including the "maximum-entropy" clustering algorithm and the $k$-means clustering algorithm was a limit case of mean shift.

Let data be a finite set $S_{D}$ (the "data", $s_{i} \in S_{D}$ ) embedded in the $N$-dimensional Euclidean space, $X$. $S_{h}(x)$ is a hypersphere of radius $h$ having the volume $h^{n} V_{n}$, centred at $x$. Let $\left.K()^{\prime}\right)$ be a 
kernel function and $w(\cdot)$ is a weight function. The sample mean with kernel $K\left({ }^{\cdot}\right)$ at $x \in X$ is defined as:

$$
m(x)=\frac{\sum_{s_{i} \in S_{h}} K\left(s_{i}-x\right) w\left(s_{i}\right) s_{i}}{\sum_{s_{i} \in S_{h}} K\left(s_{i}-x\right) w\left(s_{i}\right)}
$$

The difference $m(x)-x$ is called mean shift.

Table 1 shows the basic Mean Shift algorithm. Let $C_{Q}$ denote the clustering result data set, $c_{i} \in C_{Q}$. Define $\varepsilon$ be a pre-defined distance threshold. Let $N$ denote the number of data points $\left\{x_{i}\right\}$ in $S_{D}$. At the beginning, we need pick a seed mean randomly as the starting point of the algorithm. A simple way is that let $x_{i}$ be the centre of hypersphere $S_{h}(x)$. The core of iterative procedure in the algorithm is then applied in line 5 to line 8 . Lines from 9 to 15 check the similarity between the new cluster and the old cluster. If a new cluster is similar to an existing cluster, vote points belong to the similar cluster. On the opposite, add the new cluster to $C_{Q}$.

\section{Table 1. Pseudo code of basic procedure in Mean Shift al gorithm.}

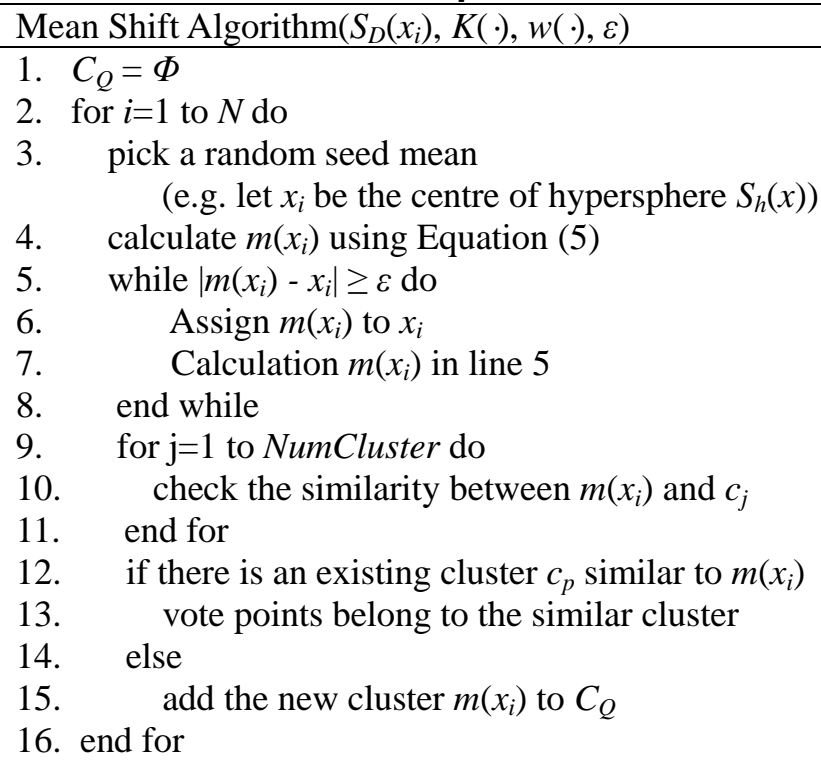

For certain distribution of points, the number of clustering was decided by clustering condition parameter finally as shown in Figure 7. In Figure 7, we randomly selected 24 points for simulation. Figure 7(a)-(c) illustrate different clustering results that are received from different $\varepsilon$.

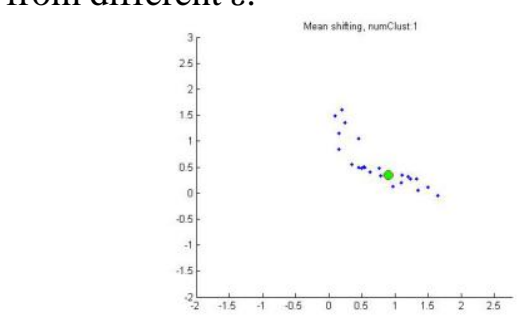

(a)

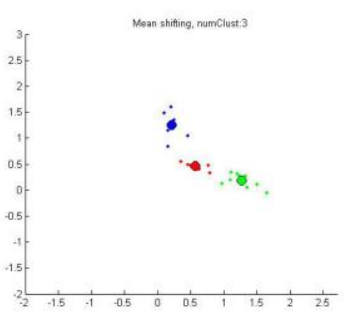

(b)

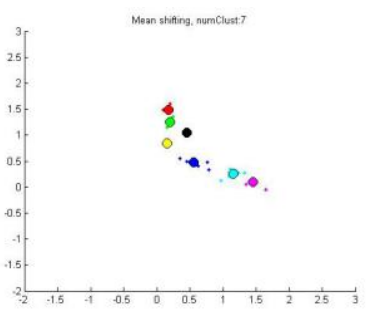

(c)

Figure 7. Different clustering results from different $\varepsilon$. (a) $\varepsilon=7.3 \times 10^{-4}$. (b) $\varepsilon=4.4 \times 10^{-4}$. (c) $\varepsilon=2.5 \times 10^{-4}$. 
Since the distribution of the laser data points is disordered at different scans. It is impractical to find an appropriate parameter $\varepsilon$ to determine the number of clusters uniformly. In the process of actual localization, the number of laser points will decrease as the distance between human and laser range finder increases. A small number of clustering points will be not enough to reflect the shape of the detection. The role of clustering will turn pale for large number of clustering points. An effective way to solve the problem above was sprung out when we amplified the detection result in Figure 6(a) as shown in Figure 8(a). To obtain appropriate number of clusters to reflect human contour, we divided the edges of detection into several areas. Each of these areas is a square. The length of square side is $10 \mathrm{~cm}$. Scan points from each area are grouped as one cluster. Figure 8(b) illustrates the clustering result. Green points are final clustering points that used to reflect human contour. One advantage of the area-divided clustering mode is that the number of final clustering points will not decrease sharply as the distance increases. Another benefit of this method is that the finalclustering points will not be affected by the overlaps of detection and increased laser points while using multiple laser range finders collaborative perception.

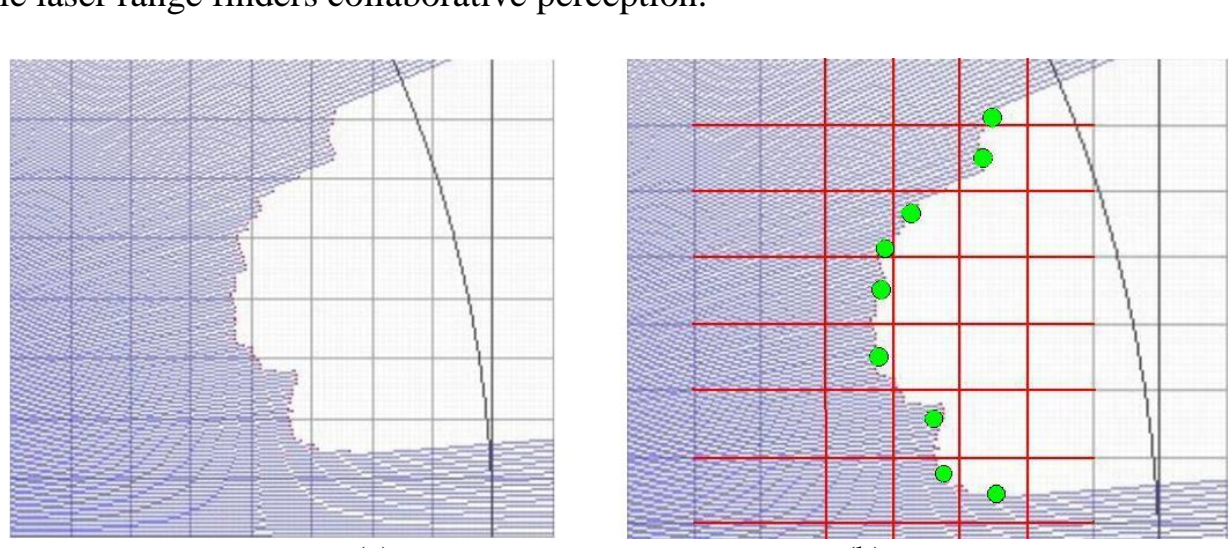

(b)

Figure 8 Amplified detection and clustering result. (a) Close-view of the detection results in figure 6(a). (b) Clustering result of area-divided mode.

\section{Cooperative Localization System}

One laser range finder is helpless for localization of multiple persons especially when occlusion occurs In this section, a system with three laser range finders distributed cooperatise localize human position is introduced. That is not to say the more laser range finders the more targets localization can be solved. The complexity of information fusion will be increased sharply when a space contains a large number of equipments tó exchange data.

\subsection{Distribution of Laser Range Finders}

In pur system, three UTM-30LX laser range finders are employed. They are distributed on three tables as shown in Figure 9. These distributed laser range finders are mounted $95 \mathrm{~cm}$ from ground. A height is chosen that the sensors could scan above lots of messy obstacles such as miniature garden or benches. Another advantage of this height is laser beams will scan on the torso of a person in the process of localization. Scan beams are spaced quite far apart (over $1.2 \mathrm{~cm}$ apart at a range of $3 \mathrm{~m}$ ) at long range. Detection of small features like legs (only about 10 scan beams is effective apart at a range of $3 \mathrm{~m}$ ) is difficult. Detection of a large target, like a torso, is more robust. 


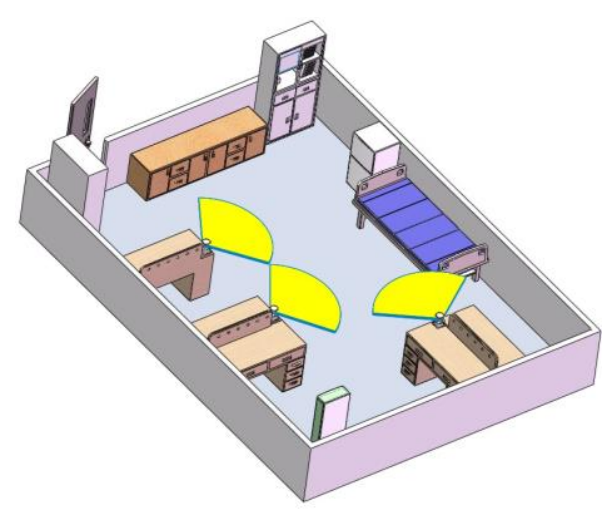

Figure 9. Distribution of laser range finders in a room.

\subsection{Sensor Calibration}

Sensors calibration plays an important role in the proposed system. Che purpose of calibration is to determine the relative positions among the lasêr range finders. We used a slender rod as a calibration reference object. The rodis about $1.5 \mathrm{~m}$ in length and 0.8 $\mathrm{cm}$ in radius. Since the diameter of the rod is $1.6 \mathrm{~cm}$, there will be at least one laser beam scan on the slender rod within $3.6 \mathrm{~m}$ (usually about four laser beams apart at a range of $1.5 \mathrm{~m}$ ). Let the position of the slender rod as the origin. We built a $2 \mathrm{D}$ plane coordinate system as shown in Figure 10. The horizontaldirection is to the west as the $x$ axis and vertical direction is to the south as the $y$ axis. The relative positions of laser range finders are calibrated off-line.

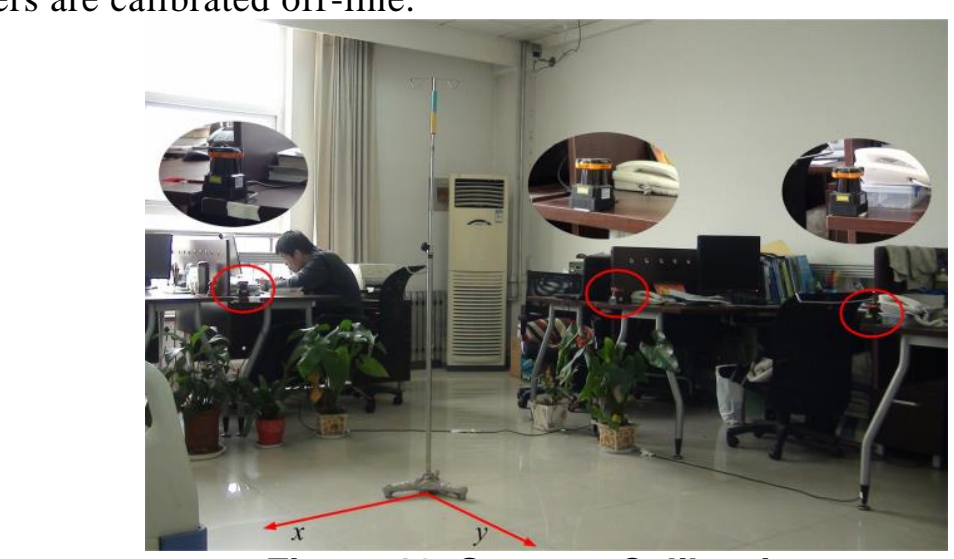

Figure 10. Sensors Calibration.

\subsection{Human Center Position Extraction}

One problem unsolved is how to utilize the limited detection information to extract the center of human eventually. As mentioned in [9] the central location of an object can be extracted by fitting the surface of the object. After clustering process based on area-divided mode, the detection contour of human can be represented by several points. Due to the contour of human body approximates ellipse, a method named incomplete ellipse fitting based on least square as mentioned in [19] is used to extract the center of the target person. Figure 11 illustrates a simulation of human center position extraction. Green points are clustering points that are used to reflect the detection contour of target people. The dotted oval is fitted by clustering characterized points. The center of the ellipse is considered as the estimation of human position. 


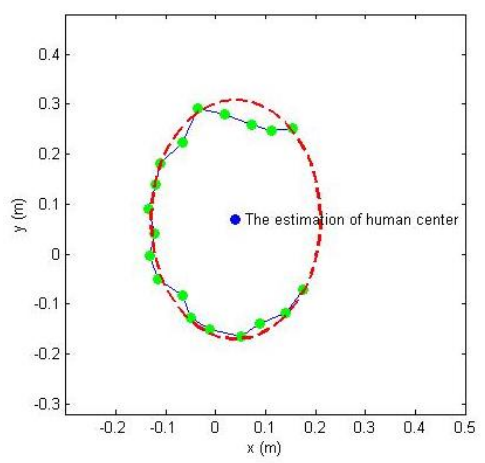

Figure 11. Center position extraction of a person.

\section{Experimental Results}

Figure 10 illustrates the actual localization experimental scene in a room. The area of the room is $6.8 \times 5.2 \mathrm{~m}^{2}$. In order to verify efficiency of the proposed method, several types of walking paths are set up and the results of localization are shown in Figure 12 and Figure 13. In each figure, the red point is the origin of coordinate system. Laser range finders are calibrated based on this point. The black lines are expected walking paths. These green, blue and pink points are localization results.

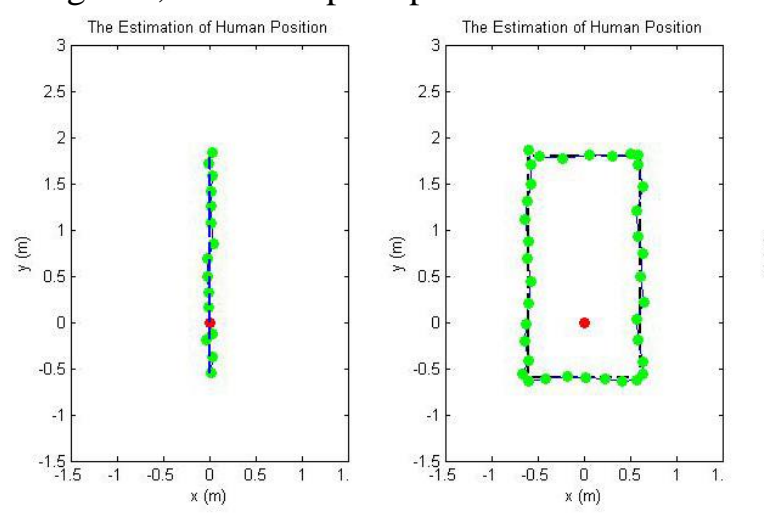

(a)

(b)

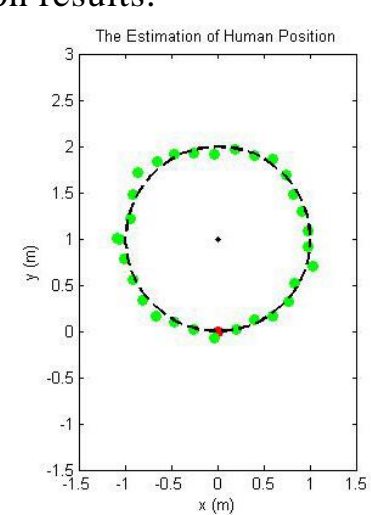

(c)

Figure 12. Localization results of one person. (a) Straight line. (b) Rectangle.

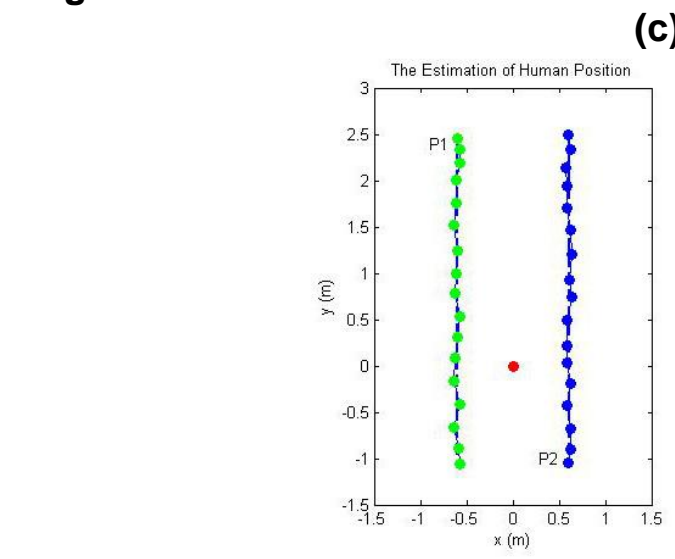

(a)

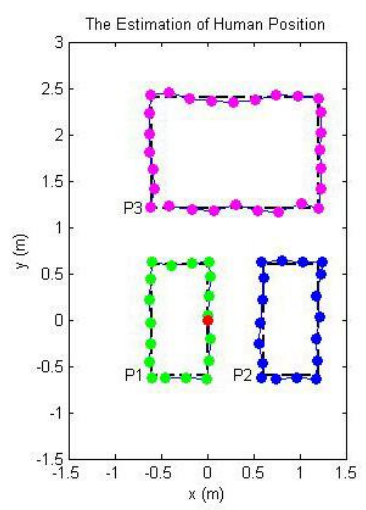

(b)

Figure 13. Localization results of multiple persons. (a) Parallel walking (face to face). (b) Walking along different rectangles. 
In Figure 12, three experiments are carried out by one person walking along three different types of paths. Figure 13 (a) shows the localization result of two person walking parallel (face to face) and Figure 13 (b) illustrates three experimenters walking along different rectangle paths at the same time. Although we can hardly ensure our experimenters walking in accordance with the preset paths strictly, the accuracy of localization is surprising from a large number of experimental results as shown in Table 2.

Table 2. Average error of localization results (cm).

\begin{tabular}{cccc}
\hline Num of people & Type of walking path & $\begin{array}{c}\text { Distributed cooperative } \\
\text { localization system }\end{array}$ & $\begin{array}{c}\text { Distributed vision } \\
\text { system }\end{array}$ \\
\hline One & Line & 5.328 & 14.63 \\
One & Rectangle & 6.613 & 18.57 \\
One & Circle & 12.376 & 28.14 \\
Two(P1, P2) & Parallel (face to face) & (P1) 5.469 & (P1) 24.15 \\
Three(P1, P2, P3) & Rectangles (different) & (P2) 5.717 & (P2) 27.82 \\
& & (P1) 7.721 & (P1) 34.68 \\
& & (P2) 8.286 & (P2) 31.96 \\
\hline
\end{tabular}

A visual localization system is also employed to measure human positionasing distributed cameras [20]. The accuracy is better than [3] which used multiple cameras too. Figure 14 shows the localization result using the distributed vision system. In Figure 14, the two small images above are collected by distributed cameras. The image in the left corner shows the localization result, the red dotted line is the desired walking path and the blue curve is the positioning trajectory. The average errof of localization is about $20 \mathrm{~cm}$. Moreover, our cooperative localization system using distributed laser range finders has showed good accuracy under the same condition.

Table 2 summarized the average errors of localization between our distributed cooperative localization system and distributed vision system. Each type of walking paths was experimented for ten times. The result of proposed method exhibited a higher accuracy than that of distributed vision system. Two reasons may contribute to these magic results. One is data process using Kalman filter guaranteed the accuracy of perceptual information. The other is the area-divided clustering mode proyided an efficient representation of detection features. Localization of maltiple persons us ng distributed vision system is more complicated. The vision localization system cam hardly afford the third person localization in Table 2 due to field-of-view overlap among hamans. Besides, the installed position of cameras is another vital factor that may leads to localization failure.

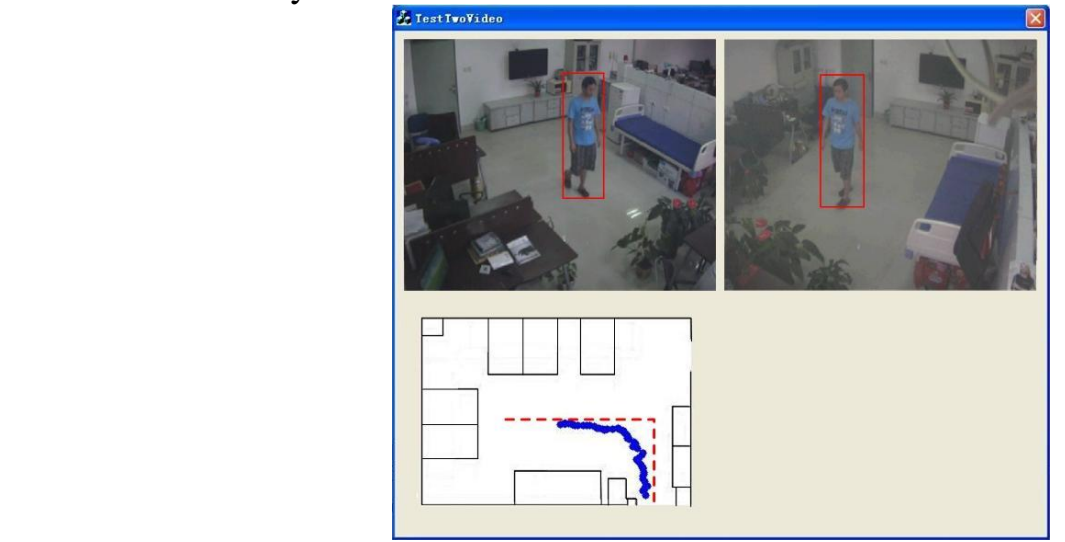

Figure 14. Localization results using distributed vision system in [20].

\section{Conclusion and Future Work}


Human position is considered as basic and useful information for service robot to implement various applications. In this paper, a method using distributed laser range finders for human localization was presented. Since raw data from laser range finder contains some small errors, the Kalman filter was employed for laser data processing. An area-divided clustering mode is introduced to group scan points to represent the contour of human. Centers of humans are estimated from the obtained clusters using incomplete ellipse fitting. The proposed method can be applied to many applications in intelligent space such as mobile robot navigation. On one hand, a mobile robot can provide fast service if the real-time position of human is known in advance. On the other hand, the walking trajectories of human will provide guidance for mobile robot.

Although the experimental results illustrate the performance and precision of localization was encouraging, the system could not identify humans. Generally, an object can be identified more directly based on color or shape characteristics from the vision information. Hence, we would like to study on the fusion of laser localization system and visual localization system in the future.

\section{ACKNOWLEDGEMENTS}

This work is supported by National Natural Science Foundation of China(61075092), Natural Science Foundation of Shandong Province (ZR2011FM011), Independent Innovation Foundation of Shandong University-IIFSDU(2011JC017).

\section{References}

[1] S. I. Lee, C. S. Jang, S. H. Kim, M. C. Ron and B. S Seo, Issues and Implementation of a URC Home Service Robot", Proceeding of the 16th IEEE Internationas Symposium on Robot and Human interactive Communication, Jeju, Korea, (2007) August, pp. 570-575.

[2] M. Kim, S. Kim, S. Park, M-T. Cho M. Kim and GA "Service Robot for the Elderly", IEEE Robotics and Automation Magazine, vol. 16, (2009), pp.

[3] J. Shen, W. Yan, P. Miller anc H. Zhou, "Human Localization in a Cluttered Space using Multiple Cameras", Proceeding of the 7th IEEE International Conference on Advanced Video and Signal Based Surveillance, Inst. of ECIT, Queen̂s Univ.Belfast Queens Island, Belfast, UK, (2010), pp. 85-90.

[4] C. R. Yu, C. L. Wu, C.H. Lu and L. C. Fu, "Human Localization via Multi-cameras and Floor Sensors in Smart Home", Proceeding of the IEEE International Conference on Systems, Man, and Cybernetics, Taipei, Taiwan, (2006), pr. 3822-3827.

[5] J. Yao and J. M Odobez, "Multi-Person Bayesian Tracking with Multiple Cameras", In book: Multi-Camera Networks: Principles and Applications, edited by Aghajan H. and Cavallaro A., Academic press (2009), pp. 363-388.

[6] T. Obo, N. Kubota-K. Taniguchi and T. Sawayama, "Human Localization based on Spiking Neural Network in Intelligent Sensor Networks", Proceeding of the 2011 IEEE Workshop on Robotic Intelligence in Informationally Structured Space (RiiSS), Tokyo Metropolitan Univ., Tokyo, Japan, (2011), pp. 125-130.

[7] Y. Yang, C. Feng, X. Guo and G. Wang, "Compressed Infrared Bearing Sensor for Human Localization: Design and Implementation", Proceeding of the IEEE International Conference on Information and Automation, Shenyang, China, (2012), pp. 936-940.

[8] D Lieckfeldt, J. You and D. Timmermann, "Exploiting RF-scatter: Human Localization with Bistatic Passive UHF RFID-systems", Proceeding of the IEEE International Conference on Wireless and Mobile Computing, Networking and Communications, Inst. of Appl. Microelectron. and Comput. Eng., Univ. of Rostock, Rostock, Germany, (2009), pp. 179-184.

[9] J. Yao, P. Taddei, M. R. Ruggeri, G. Bostrom and V. Sequeira, "Automatic Laser-based Identification for UF_6 cylinders", Machine Vision and Applications, vol. 24, (2013), pp. 305-318,

[10] H. Kim, W. Chung and Y. Yoo, "Detection and Tracking of Human Legs for a Mobile Service Robot", Proceeding of the IEEE/ASME International Conference on Advanced Intelligent Mechatronics, Montrea, Canada, (2010), pp.812-817.

[11] N. Bellotto and H. Hu, "Multisensor-based Human Detection and Tracking for Mobile Service Robots", IEEE Transactions on Systems, man, and cybernetics-part B: cybernetics, vol. 39, no. 1, (2009) February, pp. 167-181. 
[12] A. Feldman, T. Balch, M. Hybinette, and R. Cavallaro, "The Multi-ICP Tracker: An Online Algorithm for Tracking Multiple Interacting Targets”, Journal of Field Robotics, vol. 29, no. 2, (2012), pp. 258-276,

[13] H. Zhao, J. Sha, Y. Zhao, J. Xi, J. Cui, H. Zha, and R. Shibasaki, "Detection and Tracking of Moving Objects at Intersections Using a Network of Laser Scanners", IEEE Transactions on Intelligent Transportation Systems, vol. 13, no. 2, (2012) June, pp. 655-670.

[14] D. Simon, "Kalman Filtering with State Constraints: A Survey of Linear and Nonlinear Algorithms", IET Control Theory and Applications, vol. 4, no. 8, (2010), pp. 1303-1318.

[15] T. Sasaki, H. Tamura, H. Hashimoto and F. Inoue, "Position Estimation based on the Target Shape Information using Laser Range Finders for Intelligent Space", Proceeding of the IEEE/ASME International Conference on Advanced Intelligent Mechatronics, Montrea, Canada, (2010), pp. 605-610.

[16] S. Z. Selim and M. A. Ismail, "K-means-type algorithms: A Generalized Convergence Theorem and Characterization of Local Optimality", IEEE Trans. Pattern Analysis und Machine Intelligence, vol. 6, (1984), pp. 81-86.

[17] N. B. Karayiannis, "MECA: Maximum Entropy Clustering Algorithm", Proceedings of the 3th IEEE World Congress on Computational Intelligence, vol. 1, (1994), pp. 630-635.

[18] Y. Cheng, "Mean Shift, Mode Seeking, and Clustering", IEEE Transactions on Pattern Analysis and Maching Intelligence, vol. 17, no. 8, (1995) August, pp. 790-799,

[19] Y. Zou and B. Wang, "Fragmental Ellipse Fitting based on Least Square Algorithm", Chinese Journal of Scientific Instrument, vol. 27, no. 7, (2006), pp. 808-812.

[20] H. Jiang, "Vision Service Support System of Intelligent Space in the Hospital", Master's Thesis, Shandong University, (2011).

\section{Authors}

Peng Duan, he is currently pursuing his Ph.D. in School of Control Science and Engineering, Shandong University, Jinan, China He received the B.S. degree from College of Automation Engineering, Qingdao University, Qingdao, China, in 2010. His research interests include service robot navigation and distributed sensors localization system.

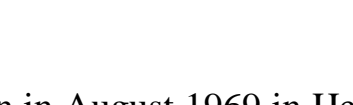

Guohui Tian, he was born in August 1969 in Hebei, China. He is a professor in School of Control Science and Engmeering, Shandong University. And also he is the member of Chinese Association for Artificial Intelligent Autonomous System. He received the B.S. degree from Department of Mathematics, Shandong University, Jinan, China, in 1990, the M.S. degree from the Department of Automation, Shandong University of Technology, Jinan, China, 1993 and the $\mathrm{Ph} \mathrm{D}$ degree from School of Automation, Northeastern University, Shenyang, China, in 1997. He studied as a post-Doctorial researcher in School of Mechanical Engineering of Shandong University from 1999 to 2001, and studied as a visiting professor in Graduate School of Eegineering of Tokyo University of Japan from 2003 to 2005. His research interests include service robot, intelligent space, collaboration and cooperation of multi-robot system.

We Zhang, he is with the School of Control Science and Engineering, Shandong University, China. His research interests include image processing, computer vision, biomedical engineering, and robotics. He has published about 30 papers in prestigious journals and refereed conferences. He served as a program committee member and reviewer for various international conferences and journals in image processing, computer vision and biomedical engineering. 
International Journal of Hybrid Information Technology

Vol.7, No.3 (2014)

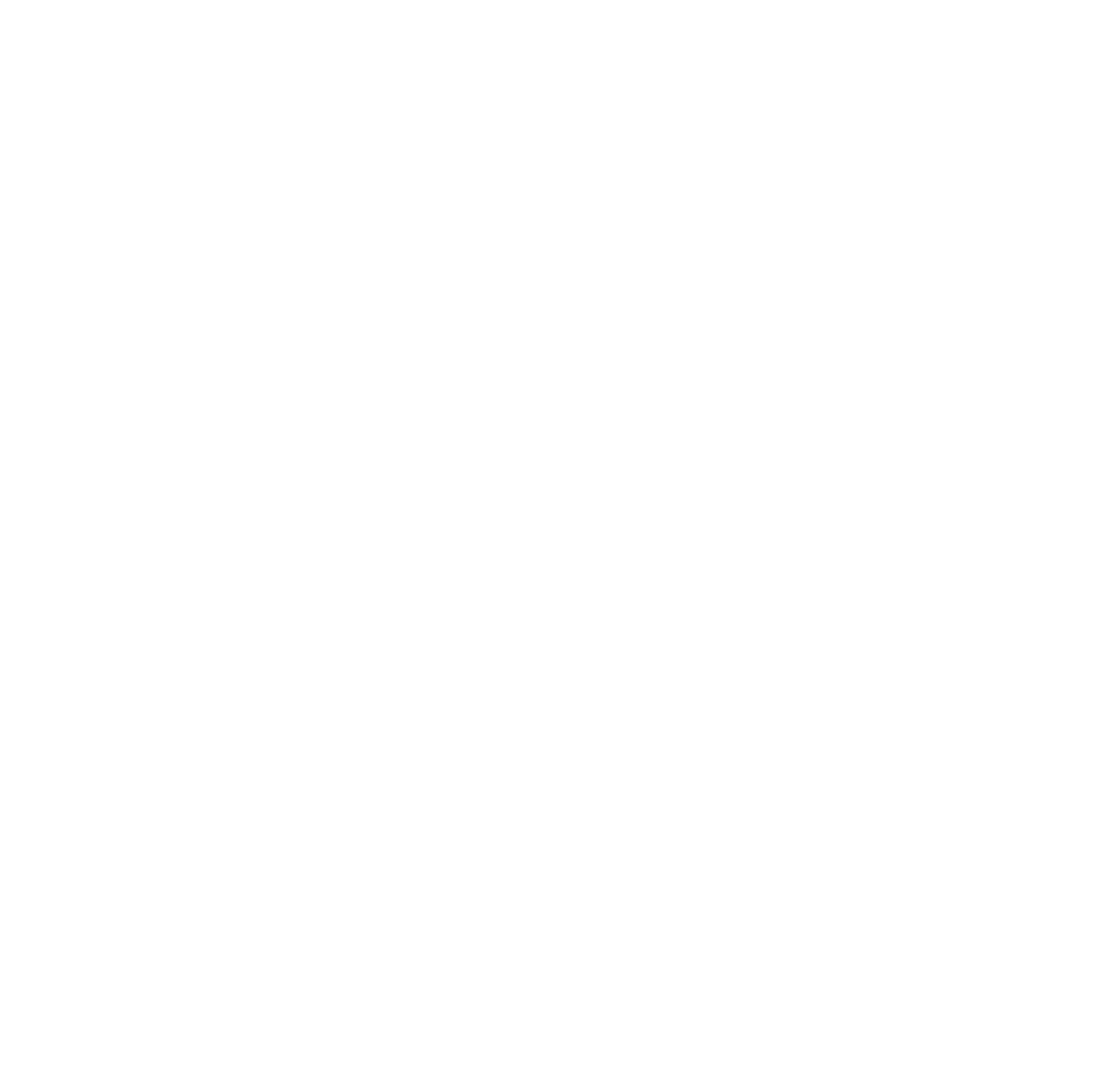

